\title{
Solar wind heating by an embedded quasi-isothermal pick-up ion fluid
}

\author{
H. J. Fahr \\ Received: 11 December 2001 - Revised: 27 May 2002 - Accepted: 4 June 2002
}

Institut für Astrophysik und Extraterrestrische Forschung der Universität Bonn Auf dem Hügel 71, D-53121 Bonn, Germany

\begin{abstract}
It is well known that the solar wind plasma consists of primary ions of solar coronal origin and secondary ions of interstellar origin. Interstellar H-atoms penetrate into the inner heliosphere and when ionized there are converted into secondary ions. These are implanted into the magnetized solar wind flow and are essentially enforced to co-move with this flow. By nonlinear interactions with wind-entrained Alfvén waves the latter are processed in the co-moving velocity space. This pick-up process, however, also causes actions back upon the original solar wind flow, leading to a deceleration, as well as a heating of the solar wind plasma. The resulting deceleration is not only due to the loading effect, but also due to the action of the pressure gradient. To calculate the latter, it is important to take into account the stochastic acceleration that suffers at their convection out of the inner heliosphere by the quasi-linear interactions with MHD turbulences. Only then can thepresently reported VOYAGER observations of solar wind decelerations and heatings in the outer heliosphere be understood in terms of the current, most likely values of interstellar gas parameters. In a consistent view of the thermodynamics of the solar wind plasma, which is composed of secondary ions and solar wind protons, we also derive that the latter are globally heated at their motion to larger solar distances. The arising heat transfer is due to the action of suprathermal ions which drive MHD waves that are partially absorbed by solar wind protons and thereby establish their observed quasi-polytropy. We obtain a quantitative expression for the solar wind proton pressure as a function of solar distance. This expression clearly shows the change from an adiabatic to a quasi-polytropic behaviour with a decreasing polytropic index at increasing distances, as has been observed by the VOYAGERS. This also allows one to calculate the average percentage of the intitial energy fed into the thermal proton energy. In a first-order evaluation of this expression we can estimate that under stationary flow conditions about $10 \%$ of the initial injection energy is eventually transfered to solar wind protons, independent of the actual injection rate.
\end{abstract}

Correspondence to: H. J. Fahr (hfahr@astro.uni-bonn.de)
Key words. Interplanetary physics (energetic particles; interstellar gas; solar wind plasma)

\section{Modulation of the solar wind flow by secondary ions}

It was determined in recent work on the dynamics of the modulated solar wind (see Holzer, 1972; Fahr, 1973; Isenberg, 1986; Baranov and Malama, 1993, 1995; Pauls et al., 1995; Zank et al., 1996a; Baranov et al., 1998; Zank, 1999; Fahr and Rucinski, 1999; Fahr et al., 2000) that ion-loading of the original solar wind enforces a deceleration and a decrease in the effective Mach number of the flow with increasing solar distances. In addition, the deceleration is also determined by the gradient of the pressure of secondary ions (in brief: $\mathrm{P}(2)^{\prime}$ s) acting upon the mixed two-fluid plasma flow. Here, suprathermal $\mathrm{P}(2)^{\prime}$ 's behave similar to a hot gas component embedded in a cold one, the solar wind protons (in brief $\left.\mathrm{P}(1)^{\text {'s }}\right)$. Representing the $\mathrm{P}(2)$ - pressure in the form $P_{2}=\alpha \rho_{2} v_{w}^{2}$ (quantities related to $\mathrm{P}(2)$ 's are indicated with the suffix " 2 ", those related to $\mathrm{P}(1)$ 's with the suffix " 1 "), as suggested by Fahr and Fichtner (1995), the following differential equation for the decelerated solar wind can be obtained (see Fahr and Rucinski, 2001):

$$
\frac{d}{d r} v_{w}=\frac{-m_{p} \beta_{e x} \frac{1+\alpha}{\rho_{1}+\rho_{2}}+\frac{2 \alpha}{r} \xi v_{w}}{1+\alpha \xi}
$$

Here, $\beta_{e x}=\sigma_{e x} n_{H} n_{1} v_{w}$ is the local $\mathrm{P}(2)$ - injection rate. The function $\xi=\rho_{2} /\left(\rho_{1}+\rho_{2}\right)$ denotes the relative abundance of $\mathrm{P}(2)^{\prime}$ 's with respect to all protons. Integration of the above differential equation then yields:

$v_{w}=v_{w 0} \exp \left[\int_{r_{0}}^{r} \frac{\frac{2 \alpha}{r} \xi-n_{H} \sigma_{e x}(1-\xi)(1+\alpha)}{1+\alpha \xi} d r\right]$.

With the expression for $P_{2}=(1 / 3) \rho_{2} v_{w}^{2}$ derived by Fahr and Fichtner (1995), one obtains:

$v_{w}=v_{w 0} \exp \left[\int_{r_{0}}^{r} \frac{2}{3+\xi}\left(\frac{\xi}{r}-2 n_{H} \sigma_{e x}(1-\xi)\right) d r\right]$. 
It is evident that an accurate expression for $P_{2}$ can only be derived with the knowledge of the $\mathrm{P}(2)$-distribution function $f_{2}$. This function has to be obtained as a solution of the $\mathrm{P}(2)$ - transport equation, including the effects of convection, adiabatic deceleration, and energy diffusion by Fermi-2 acceleration.

An expression for $f_{2}$, taking into account the realistic $\mathrm{P}(2)$ injection and the above-mentioned consecutive $\mathrm{P}(2)$-phasespace transport, has been obtained by Chalov et al. (1995, 1997), and as seen in Fahr and Lay (2000), can be very nicely represented by the following analytical formula:

$f_{2}=\Pi\left(x^{-0.33}\right) w^{\beta} \exp \left[-C(x)\left(w-w_{0}\right)^{\kappa}\right]$,

where $\Pi$ is a constant, $x=r / r_{E}$ is the radial solar distance in units of $\mathrm{AU}$, and $w=\left(v / v_{w}\right)^{2}$ is the squared $\mathrm{P}(2)$ velocity normalized with $v_{w}$ and $w_{0}$ being a typical injection value. Furthermore, the quantities $\beta, \kappa$, and $C(x)$ are found from a best-fit procedure with: $\beta=-\frac{1}{6} ; \kappa=\frac{2}{3}$; and: $C(x)=$ $0.442 x^{0.2}$. These results are obtained on the basis of some assumptions on amplitude and spectral slope of the Alfvénic turbulences interacting with $\mathrm{P}(2)^{\text {'s. }}$. According to the WKB theory of dissipationless Alfvén turbulence, the amplitudes are assumed to fall off with distance by $x^{-3}$, and the spectral power index was taken to be $\gamma_{k}=5 / 3$.

With the above Eq. (4) for $f_{2}$, one then obtains the $\mathrm{P}(2)$ density by:

$n_{2}=2 \pi \Pi x^{-0.33}\left[\frac{3}{2} C(x)^{-2} \Gamma(2)\right]$,

and the $\mathrm{P}(2)$-pressure by:

$P_{2}=\frac{2 \pi}{3} \Pi x^{-0.33}\left(\frac{1}{2} m_{p} v_{w}^{2}\right)\left[\frac{3}{2} C(x)^{-\frac{7}{2}} \Gamma\left(\frac{7}{2}\right)\right]$,

where $\Gamma(y)$ is the Gamma function for the argument $y$. Equations (5) and (6) then lead to the following expression for $P_{2}=P_{2}\left(\rho_{2}\right)$ :

$P_{2}=\frac{5}{16} \sqrt[2]{\pi} C(x)^{-\frac{3}{2}} \rho_{2} v_{w}^{2}=\alpha(x) \rho_{2} v_{w}^{2}$,

showing that with the above expression for $P_{2}(x)$, one obtains a function $\alpha(x)=1.83 x^{-0.3}$ with decreasing values for $\alpha(x)$ for increasing solar distances $x$. This shows that obviously at larger distances the adiabatic deceleration starts to slowly overcompensate for the effect of wave-driven Fermi-2 accelerations. The above formula in view of the results used from Fahr and Lay (2000) should be valid at distances of $x \geq x_{c}=15$, where $\alpha=\alpha_{c}=\alpha\left(x_{c}\right)$ evaluates to $\alpha_{c}=0.44$. In the Appendix, we show on the basis of an approximative evaluation of Eq. (7) how the pressure $P_{2}(x)$ and the function $C_{2}(x)=P_{2}(x) / \rho_{2}(x)$ behave with solar distance $x$, suggesting the approximation $C_{2}(x)=C_{2}=$ const.

\section{Thermodynamics of the $\mathbf{P}(1)-\mathbf{P}(2)$ two-fluid solar wind}

$\mathrm{P}(2)$ 's are produced by ionization of interstellar neutral atoms in the heliosphere and are convected outwards with the solar wind flow as a separate suprathermal ion fluid. The thermodynamic behaviour of this "hot" fluid at its motion outwards to the outer heliosphere, until now, is not completely understood. As one clearly knows $\mathrm{P}(2)$ 's drive waves by virtue of their distribution function which is unstable with respect to the excitation of wave power (see, for example, Wu and Davidson, 1972; Hartle and Wu, 1973; Lee and Ip, 1987; Freund and Wu, 1989; Fahr and Ziemkiewicz, 1988; Gray et al., 1996), but they themselves also undergo Fermi-2 energization (energy diffusion) by nonlinear wave-particle interaction with already preexisting, convected wave turbulences (see, for example, Bogdan et al., 1991; Chalov et al., 1995, 1997; le Roux and Fichtner, 1997).

In the following we want to study the branching of the relevant energy flows and thereby try to respect the observational fact that $\mathrm{P}(1)^{\prime}$ s behave non-adiabatic, but polytropic at their expansion to large solar distances (see Whang, 1998, Whang et al., 1999). This evidently expresses the fact that solar wind protons are globally and continuously heated at their motion to larger solar distances. This global heating cannot be related to sporadic events, such as the passages of corotating interaction regions (CIR's) or solar eruptive events (see also Fisk et al., 2000). In contrast, it appears highly likely to be caused by $\mathrm{P}(2)$ 's, which drive MHD waves that are partially re-absorbed by solar wind protons $\mathrm{P}(1)^{\prime}$ s.

Already Parker (1964) and Coleman (1968) expected that some extended heating due to dissipation of waves might cause a non-adiabatic expansion of the solar wind beyond its critical point. This non-adiabatic solar wind temperature behaviour, meanwhile, in fact is clearly recognized in the data taken by the VOYAGER-1/2 spacecraft (see Richardson et al., 1995; Whang, 1998; Whang et al., 1999). The dissipation of non-Alfvénic turbulence energy to solar wind protons was then more quantitatively estimated by Matthaeus et al. (1994) to take place with a rate $q_{t u r b} \simeq \rho_{s} u^{3} / l$, where $\rho_{s}, u, l$ are the solar wind mass density, the rms turbulent fluctuation speed, and the turbulent correlation scale.

In order to find out more about the dependence of solar distance $r$ on these quantities $u$ and $l$, Zank et al. (1996b) studied the evolution of low-frequency turbulence power in the solar wind on the basis of a scale-separated equation developed by Zhou and Matthaeus (1990), describing the evolution of amplitude fluctuations $u$ and $b$ about the mean velocity $V_{w}$ and the mean magnetic field $\boldsymbol{B}$. In this equation for the frequency-averaged fluctuation power, these authors took into account nonlinear dissipation terms and power sources. Amongst the latter they discussed terms due to wave-driving by velocity shears and compressional effects associated with solar wind interaction regions due to pick-up ions injected into unstable distribution functions. In the solutions for $u^{2}(r)$ and $l(r)$, they could demonstrate that the usual WKB approximations are far from what can realistically be expected in 
the solar wind at large distances. Concerning far-off solar wind interaction regions at higher heliographic latitudes, one should not expect to find shear-induced turbulent energy, but outside of the so-called ionization cavity, nevertheless, one should find pick-up ion induced turbulent energy and correlation lengths $l(r)$ which from 5 AU outwards systematically decrease with distance.

Based on these results, Smith et al. (2001) also analysed the heating of the distant solar wind due to dissipation of wave turbulent energy to protons. They solved a system of coupled differential equations, describing the evolution with distance of the mean turbulent energy $u^{2}$, the correlation length $l$, and the proton temperature. The nonlinear dissipative loss term in the equation for $u^{2}$ was at the same time taken, of course with the opposite sign, as an energy gain term for the protons. Comparison of the results with VOYAGER data seem to show that, though the main tendencies can be explained by this theoretical approach, nevertheless, the predicted values for both $u^{2}$ and the solar proton temperature $T$ are fairly on the low side of the VOYAGER-2 data. This may be partly due to the mixing of high- and lowvelocity solar wind, and partly due to the fact that adiabatically cooled pick-up ions copopulate the $\mathrm{P}(1)$-Maxwell tails.

Permanent dissipation of turbulent wave power upon heating the expanding solar wind should quickly lead to a complete consumption of all convected turbulence power, unless some turbulence generating processes are operating. In this respect, Lee and Ip (1987) and Fahr and Ziemkiewicz (1988) have already indicated that $\mathrm{P}(2)^{\text {'s }}$ implanted into the expanding solar wind, by means of their unstable distribution functions, generate wave powers which can partially be reabsorbed by $\mathrm{P}(1)^{\prime}$ 's. Using quasi-linear wave-particle interaction theories by Kennel and Engelmann (1966), Gary and Feldman (1978) and Winske and Leroy (1984), the latter authors could show that under optimized conditions, up to $50 \%$ of the initial $\mathrm{P}(2)$-energy can be forwarded to $\mathrm{P}(1)^{\prime}$ s by means of $\mathrm{P}(2)$-driven waves. More recently, Williams et al. (1995) and Gray et al. (1996) have looked into this problem again. Williams et al. (1995) have given representations for the non-adiabatic expansion of the distant solar wind due to dissipation of $\mathrm{P}(2)$-driven waves within a simplified energy dissipation concept. Gray et al. (1996), within a hybrid plasma simulation code, studied the energy transfer in a homogeneous plasma background from the original unstable $\mathrm{P}(2)$ ring distribution to the $\mathrm{P}(1)$ thermal energy degree perpendicular to the magnetic field and found that for vanishing pitch-angle diffusion - at most favourable conditions like "low Beta" plasmas - about $20 \%$ of the initial $\mathrm{P}(2)$ ring energy can be handed over to $\mathrm{P}(1)^{\prime}$ 's.

In all concepts mentioned so far, however, a quantitative number for the average fraction of initial $\mathrm{P}(2)$ - energy transfered under general conditions to $\mathrm{P}(1)^{\prime}$ 's while moving towards the heliospheric termination shock, including pitchangle diffusion and general forms of nonlinear wave-particle couplings, could not be given. Here we may gain insight from the observational result presented by Whang (1998) or Whang et al. (1999), showing that the distant P(1)'s behave polytropic with a best-fitting polytropic index of $\gamma^{*}=1.28$. Since $\gamma^{*}$ turns out to be substantially smaller than the adiabatic index $\gamma=5 / 3 \simeq 1.667$, it is evident that some continuous, i.e. non-CIR-correlated heating of $\mathrm{P}(1)^{\prime}$ 's takes place which outside of the ionization cavity, may be ascribed to the action of $\mathrm{P}(2)$ 's. This $\mathrm{P}(1)$-heating, since global in its nature and independent on latitude, most certainly must be due to wave energy that is continuously coupled from the $\mathrm{P}(2)^{\prime}$ 's via feeding of wave turbulences to the $\mathrm{P}(1)^{\prime}$ s, due to nonlinear or quasi-linear wave-particle couplings (see Williams et al., 1995; Zanket al., 1996). Thus, represents an energy sink for the $\mathrm{P}(2)^{\prime} \mathrm{s}$ as they pump energy into wave turbulent power, but at the same time it may again also partially represent an energy source for $\mathrm{P}(1)^{\prime}$ s, which reabsorb parts of these turbulences undergoing energy diffusion.

Here, on the one hand, we would like to respect the fact that $\mathrm{P}(2)^{\prime}$ s undergo a type of Fermi-2 acceleration or transittime damping process, which is clearly manifest as an ubiquitous heliospheric phenomenon, both in view of theory and observations (e.g. see Fisk et al., 2000). But on the other hand, we have to take into account that these $\mathrm{P}(2)^{\prime}$ 's that initially drive wave turbulences also experience genuine energy losses. This needs to be taken into account by a complete $\mathrm{P}(2)$ thermodynamics. These $\mathrm{P}(2)$ energy losses are primarily due to the generation of wave power which eventually is absorbed by protons, as discussed by Huddleston and Johnstone (1992) or Zanket al. (1996b). In addition, some loss of $\mathrm{P}(2)$ energy in a more hydrodynamic view is also connected with the work done by $\mathrm{P}(2)^{\prime}$ 's through their pressure at driving the effective solar wind with an effective bulk velocity $v_{w}$, jointly shared by $\mathrm{P}(2)^{\prime}$ s and $\mathrm{P}(1)^{\prime}$ 's (see e.g. Fahr and Rucinski, 1999).

Here we start out our considerations of the $\mathrm{P}(1)-\mathrm{P}(2)-$ two fluid thermodynamics from the earlier kinetic result obtained from Chalov and Fahr (1995) leading to a distribution function $f_{2}$, which yields the $\mathrm{P}(2)$-pressure as its third moment by the expression (see Eq. 7):

$P_{2}(r)=\alpha_{c}\left(r_{s} / r\right)^{0.3} \cdot \rho_{2} v_{w}^{2}=\rho_{2} C_{2}(r)$.

Taking this result derived from kinetic $\mathrm{P}(2)$ - studies carried out by Chalov et al. $(1995,1997)$ and supported by the results of le Roux and Fichtner (1997) as a serious physical hint, it then demonstrates that the $\mathrm{P}(2)^{\prime}$ s, in view of a nearly constant asymptotic solar wind velocity $v_{w} \simeq v_{w 0}$, essentially behave like an isothermal fluid, since with $C_{2}(r)=C_{2}=$ const., according to the relation $P_{2} / \rho_{2}^{\gamma_{2}} \simeq C_{2}$ with $\gamma_{2} \simeq 1$, one then simply derives from Eq. (8) that:

$\frac{\partial P_{2}}{\partial \rho_{2}} \simeq \frac{P_{2}}{\rho_{2}} \simeq C_{2}=K T_{2} / m_{p}$.

In the appendix we shall investigate in more detail the exact behaviour of the temperature $T_{2}(r)$ and shall demonstrate how well the above approximation is fulfilled. In the following, however, we shall make use of Eq. (8). In hydrodynamical terms this equation means that $\mathrm{P}(2)^{\prime}$ 's, when expanding with the solar wind, experience just enough heating to keep 
their temperature $T_{2}$ about constant at the expansion of the solar wind to larger distances. This phenomenon must thus be reflected in a fine-tuned strength of the energy input terms on the RHS of the equation of conservation of the $\mathrm{P}(2)-$ enthalpy flow given by:

$$
\begin{gathered}
\operatorname{div}\left(\frac{\gamma}{\gamma-1} P_{2} \boldsymbol{v}_{w}\right)-\left(\boldsymbol{v}_{w} \circ \nabla\right) P_{2} \\
=\beta_{e x}\left(\frac{1}{2} m_{p} v_{w}^{2}\right)+Q_{2}
\end{gathered}
$$

where $\beta_{\text {ex }}$ is the $\mathrm{P}(2)$ - injection rate, $E_{i}=\frac{1}{2} m_{p} v_{w}^{2}$ is the initial $\mathrm{P}(2)$ injection energy seen in the solar wind rest frame, and $Q_{2}$ denotes the net energy input into the $\mathrm{P}(2)$ - fluid due to nonlinear wave-particle interactions, including losses due to wave-driving and gains due to Fermi-2 accelerations.

We now want to find the form of the unknown term $Q_{2}$ that can satisfy the above differential equation10. Remembering that the $\mathrm{P}(2)$ - mass flow conservation requires:

$m_{p} \beta_{e x}=\operatorname{div}\left(\rho_{2} \boldsymbol{v}_{w}\right)$

we then obtain:

$\operatorname{div}\left(\left(\frac{\gamma}{\gamma-1}-\frac{v_{w}^{2}}{2 C_{2}}\right) \rho_{2} \boldsymbol{v}_{w}\right)-\left(\boldsymbol{v}_{w} \circ \nabla\right) \rho_{2}=Q_{2} / C_{2}$

and can derive the following result:

$Q_{2}=\left(\frac{\gamma}{\gamma-1}-\frac{v_{w}^{2}}{2 C_{2}}\right) \operatorname{div}\left(P_{2} \boldsymbol{v}_{w}\right)-\left(\boldsymbol{v}_{w} \circ \nabla\right) P_{2}$.

The pick-up ion fluid gains the initial injection energy $E_{i}$ per creation of new pick-up's and by energy diffusion processes due to nonlinear wave-particle interactions; but it also loses thermal energy by adiabatic cooling and by driving wave power with the unstable parts of its distribution function $f_{2}$. The source $Q_{2}$ only comprises the net balance of energies pumped into the wave turbulences by kinetic instabilities and absorbed from the wave turbulences by energy diffusion. Hence, $Q_{2}$ is the net energy lost by pick-up's and finally mediated by waves to solar wind protons. Thus, with the above Eq. (13) we have just found the form of a net energy input $Q_{2}$ that leads to an isothermal $\mathrm{P}(2)$ - behaviour.

Before we study the thermodynamics of the solar wind protons separately, we take a look into the thermodynamics of the joint $\mathrm{P}(1)-\mathrm{P}(2)$ - two-fluid system, which is formulated by:

$$
\begin{gathered}
\operatorname{div}\left(\frac{\gamma}{\gamma-1}\left(P_{2}+P_{1}\right) \boldsymbol{v}_{w}\right)-\left(\boldsymbol{v}_{w} \circ \nabla\right)\left(P_{2}+P_{1}\right) \\
\quad=\beta_{e x}\left(\frac{1}{2} m_{p} v_{w}^{2}-K T_{1}\right)+Q_{2}+Q_{1}
\end{gathered}
$$

What is now required is what is physically reasonable for a stationary outflow. This two-fluid system, in the absence of any external energy sources aside from the evident energy sinks, is connected per creation of $\mathrm{P}(2)$, with the removal of thermal $\mathrm{P}(1)$-energy, i.e. $K T_{1}$, and the gain of the $\mathrm{P}(2)$-injection energy, i.e. $E_{i}$. This then leads to the obvious conclusion that the energy inputs $Q_{1}$ and $Q_{2}$ to the $\mathrm{P}(1)$ and the $\mathrm{P}(2)$ - fluids, respectively, connected with nonlinear wave-particle interactions, have to cancel each other (i.e. no net energy gain or loss of the wave fields is expected!). This then evidently requires that:

$Q_{1}=-Q_{2}$.

Based on this result and on the expression we have derived for $Q_{2}$ in Eq. (13), we thus obtain the single-fluid thermodynamics of $\mathrm{P}(1)^{\prime}$ s given by the following equation:

$$
\begin{gathered}
\operatorname{div}\left(\frac{\gamma}{\gamma-1} P_{1} \boldsymbol{v}_{w}\right)-\left(\boldsymbol{v}_{w} \circ \nabla\right) P_{1}=-\beta_{e x}\left(K T_{1}\right) \\
-\left(\frac{\gamma}{\gamma-1}-\frac{v_{w}^{2}}{2 C_{2}}\right) \operatorname{div}\left(P_{2} \boldsymbol{v}_{w}\right)+\left(\boldsymbol{v}_{w} \cdot \nabla\right) P_{2} .
\end{gathered}
$$

We now try to obtain from the above equation a solution for the solar wind pressure $P_{1}$ and for that purpose the arrange Eq. (16) into the more appropriate following form, keeping in mind that $P_{2}=C_{2} \rho_{2}$ (see Eq. 8) and that $\operatorname{div}\left(n_{2} \boldsymbol{v}_{\boldsymbol{w}}\right)=$ $-\operatorname{div}\left(n_{1} \boldsymbol{v}_{\boldsymbol{w}}\right)$ :

$$
\begin{aligned}
& \operatorname{div}\left(\frac{\gamma}{\gamma-1} P_{1} \boldsymbol{v}_{w}\right)-\left(\boldsymbol{v}_{w} \circ \nabla\right) P_{1}=K T_{1} \operatorname{div}\left(n_{1} \boldsymbol{v}_{\boldsymbol{w}}\right) \\
& \quad+\left(\frac{\gamma}{\gamma-1} m_{p} C_{2}-\frac{m_{p} v_{w}^{2}}{2}\right) \operatorname{div}\left(n_{1} \boldsymbol{v}_{w}\right) \\
& \quad+m_{p} C_{2}\left(\boldsymbol{v}_{w} \circ \nabla\right) n_{2} .
\end{aligned}
$$

We shall now evaluate this equation for a spherically symmetric solar wind flow assuming that (see Appendix):

$$
\frac{2 v_{w}}{r} \gg \frac{d v_{w}}{d r}
$$

can be used as a satisfactory approximation and with that obtain:

$$
\begin{aligned}
\frac{\gamma}{\gamma-1}\left[\frac{d P_{1}}{d r}+\frac{2 P_{1}}{r}\right]-\frac{d P_{1}}{d r} & {\left[-k T_{1}+\frac{\gamma}{\gamma-1} m_{p} C_{2}-\frac{m_{p} v_{w}^{2}}{2}\right] } \\
= & {\left[\frac{d n_{1}}{d r}+\frac{2 n_{1}}{r}\right]-m_{p} C_{2}\left[\frac{d n_{1}}{d r}+\frac{2}{r}\left(n_{1}+n_{2}\right)\right] . }
\end{aligned}
$$

This equation can be simplified into the following form:

$$
\begin{aligned}
& \frac{1}{\gamma-1} \frac{d P_{1}}{d r}+\frac{2 \gamma}{\gamma-1} \frac{P_{1}}{r} \\
&= {\left[-k T_{1}+\frac{1}{\gamma-1} m_{p} C_{2}-\frac{m_{p} v_{w}^{2}}{2}\right] } \\
& \cdot\left(\frac{d n_{1}}{d r}+\frac{2 n_{1}}{r}\right)-m_{p} C_{2}\left[\frac{2}{r} n_{2}\right] .
\end{aligned}
$$


Keeping in mind that $K T_{1} \ll m_{p} C_{2}=K T_{2}$, and that the $\mathrm{P}(2)$ - density is related to the total proton density by $n_{2}=$ $n-n_{1}$, with $n$ as the total solar proton density given by: $n=n_{0}\left(r / r_{0}\right)^{-2}$, then yields the following equation:

$$
\begin{aligned}
& \frac{d P_{1}}{d r}+2 \gamma \frac{P_{1}}{r}=-\left[K T_{2}-(\gamma-1) \frac{m_{p} v_{w}^{2}}{2}\right] \frac{\beta_{e x}}{v_{w}} \\
& -K T_{2}(\gamma-1)\left[\frac{2}{r}\left(n-n_{1}\right)\right]
\end{aligned}
$$

which finally, with the $\mathrm{P}(2)$-injection rate $\beta_{e x}=n_{1} n_{H} \sigma_{e x} v_{w}$, yields:

$$
\begin{aligned}
\frac{d P_{1}}{d r} & +2 \gamma \frac{P_{1}}{r}=\left[\frac{2}{r}(\gamma-1) K T_{2}\right. \\
& \left.-\left(K T_{2}+(\gamma-1) \frac{m_{p} v_{w}^{2}}{2}\right) n_{H} \sigma_{e x}\right] n_{1} \\
& -K T_{2}(\gamma-1) \frac{2 n_{0}}{r_{0}}\left(\frac{r_{0}}{r}\right)^{3} .
\end{aligned}
$$

This differential equation is of the following formal form:

$$
\frac{d P_{1}}{d r}+g_{1}(r) P_{1}=g_{2}(r)
$$

and thus has the solution:

$$
\begin{aligned}
P_{1}=\exp \left(-2 \gamma \int_{r_{0}}^{r} \frac{d r}{r}\right) \\
\left\{P_{1,0}+\int_{r_{0}}^{r} \exp \left(+2 \gamma \int_{r_{0}}^{r} \frac{d r}{r}\right) g_{2}\left(r^{\prime}\right) d r^{\prime}\right\} .
\end{aligned}
$$

Equation (23) further simplifies to:

$$
P_{1}=\left(\frac{r}{r_{0}}\right)^{-2 \gamma}\left(P_{1,0}+\int_{r_{0}}^{r}\left(\frac{r}{r_{0}}\right)^{2 \gamma} g_{2}\left(r^{\prime}\right) d r^{\prime}\right) .
$$

Representing the function $g_{2}(r)$ in the form:

$$
g_{2}(r)=g_{21}(r)+g_{22}(r)+g_{23}(r)
$$

then leads to the following solution for $P_{1}$ :

$$
P_{1}=\left(\frac{r}{r_{0}}\right)^{-2 \gamma}\left(P_{1,0}+I_{21}+I_{22}+I_{23}\right),
$$

where the integrals $I_{21}, I_{22}, I_{23}$ are given by:

$$
\begin{aligned}
& I_{21}=2 n_{0}\left(K T_{2}\right)(\gamma-1) \\
& \int_{1}^{x} x^{2 \gamma-3} \exp \left(-\Lambda\left(x^{\prime}-1\right)\right) d x^{\prime} \\
& I_{22}=\Lambda\left(K T_{2}-(\gamma-1) \frac{m_{p} v_{w}^{2}}{2}\right) n_{0} \\
& \quad \int_{1}^{x} x^{2 \gamma-2} \exp \left(-\Lambda\left(x^{\prime}-1\right)\right) d x^{\prime}
\end{aligned}
$$

$I_{23}=-K T_{2}(\gamma-1) 2 n_{0} \int_{1}^{x} x^{2 \gamma-3} d x^{\prime}$

To derive the above integrals in these forms, the density $n_{1}$, given by (see Fahr and Rucinski, 1999) was used,

$n_{1}=n_{0} x^{-2} \exp (-\Lambda(x-1))$.

Furthermore, it is assumed that the H-atom density in the outer heliosphere is essentially constant, i.e. $n_{H} \simeq n_{H 0}$, and the following abbreviations were used:

$x=r / r_{o} ; \quad$ and $\Lambda=n_{H 0} \sigma_{e x} r_{o}$.

Keeping in mind that $\Lambda=n_{H 0} \sigma_{e x} r_{0}$ is of the order of $10^{-3}$, may permit us to set in the integrals above: $\exp (-\Lambda(x-1)) \simeq 1$. In this physically reasonable approximation, one then obtains the following solution for $P_{1}$ :

$$
\begin{aligned}
P_{1} & =x^{-2 \gamma}\left[P_{1,0}+\Lambda\left(K T_{2}\right.\right. \\
& \left.\left.-(\gamma-1) \frac{m_{p} v_{w}^{2}}{2}\right) \frac{n_{0}}{2 \gamma-1}\left(x^{2 \gamma-1}-1\right)\right] .
\end{aligned}
$$

First, we now intend to investigate the polytropic behaviour of the $\mathrm{P}(2)$-heated solar wind and for that purpose, we study the expression derivable for the local polytropic in$\operatorname{dex} \gamma_{1}$ :

$\gamma_{1}=\frac{\rho_{1}}{P_{1}} \frac{d P_{1}}{d \rho_{1}}$

To evaluate Eq. (32), we first take the derivative of $P_{1}$ with respect to $r$, given in the form:

$\frac{d P_{1}}{d r}=\frac{1}{r_{0}}\left\{-\frac{2 \gamma P_{1}}{x}+P_{1,0} \Lambda\left(\alpha_{1}-\alpha_{2}\right) x^{-2}\right\}$,

where $\alpha_{1}$ and $\alpha_{2}$ are defined by:

$$
\alpha_{1}=\frac{n_{0} K T_{2}}{P_{1,0}}=\left(T_{2} / T_{1,0}\right) ; \text { and } \quad \alpha_{2}=(\gamma-1) \frac{n_{0} m_{p} v_{w}^{2}}{2 P_{1,0}}
$$

With Eq. (31) and the relation: $\frac{d \rho_{1}}{d r}=-2 \frac{\rho_{1}}{r}$, we then obtain from Eq. (32):

$\gamma_{s}(x)=\frac{\rho_{1}}{P_{1}} \frac{d P_{1}}{d \rho_{1}}=\gamma-\frac{P_{1,0}}{P_{1}} \frac{\Lambda}{4}\left(\alpha_{1}-\alpha_{2}\right) x^{-1}$.

In the following we shall demonstrate the results of the thermodynamic behaviour of $\mathrm{P}(2)$-heated $\mathrm{P}(1)$ 's by plotting in Figs. 1 to 3 the quantities $\log \left(P_{1}\right)$ versus $\log \left(\rho_{1}\right)$ with $\Delta \alpha=\alpha_{1}-\alpha_{2}, P_{1,0}$, and $\Lambda$, respectively, as open parameters.

First, in Fig. 1, the parameter $\Delta \alpha$ is varied with the following values selected: $\Delta \alpha_{1}=50 ; \Delta \alpha_{2}=30 ; \Delta \alpha_{3}=10$. As is evident in this figure the pressure $P_{1}$ drops the least with the density $\rho_{1}$, or equivalently the solar distance $x$, the higher the value for $\Delta \alpha$ is, i.e. the more efficient the $\mathrm{P}(2)$ induced heating of the $\mathrm{P}(1)^{\prime} \mathrm{s}$ is. On the other hand, in Fig. 2, we can show that when keeping the same value for $\Delta \alpha$, then the pressure $P_{1,0}$ just acts as a factor in the Eq. (31) for $P_{1}$ and hence, its variation simply moves up or down the whole 


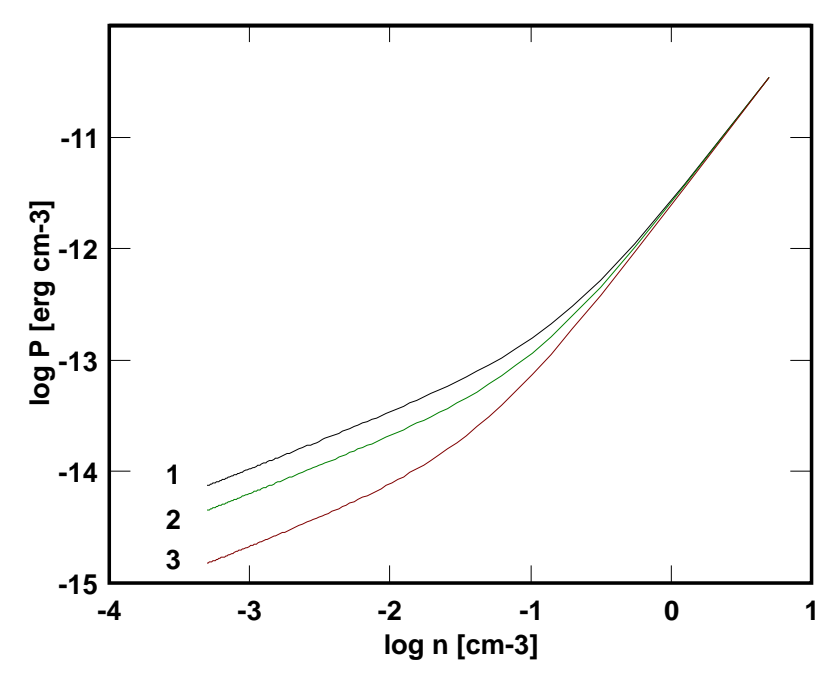

Fig. 1. Plotted is the logarithm of the solar wind pressure $P_{1}$ versus the logarithm of the density $\mathrm{n}_{1}$ at $\Lambda=1 \cdot 10^{-3}$ and $T_{1,0}=5 \cdot 10^{4} \mathrm{~K}$ for different values of $\Delta \alpha=\alpha_{1}-\alpha_{2}$, i.e. for $1: \Delta \alpha=50,2: \Delta \alpha=$ $30,3: \Delta \alpha=10$.

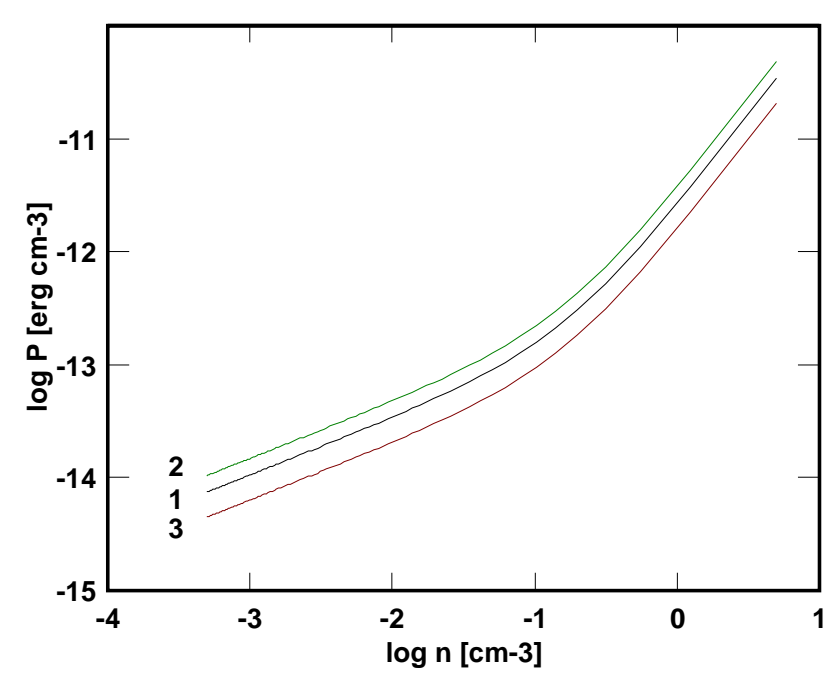

Fig. 2. Plotted is the logarithm of the solar wind pressure $P_{1}$ versus the logarithm of the density $\mathrm{n}_{1}$ at $\Lambda=1 \cdot 10^{-3}$ and $\Delta \alpha=50$ for different values of $T_{1,0}$, i.e. for $1: T_{1,0}=5 \cdot 10^{4} \mathrm{~K}, 2: T_{1,0}=$ $7 \cdot 10^{4} \mathrm{~K}, 3: T_{1,0}=3 \cdot 10^{4} \mathrm{~K}$.

curve by a constant vertical shift. The pressure $P_{1}$ at larger solar distances reacts even more sensitively to a variation in the quantity $\Lambda=n_{H} \sigma_{e x} r_{0}$. Ascribing this variation in $\Lambda$ $\left(\Lambda_{1}=1 \cdot 10^{-3}, \Lambda_{2}=2 \cdot 10^{-3}, \Lambda_{3}=3 \cdot 10^{-3}\right)$ to a corresponding variation in the $\mathrm{H}$-atom density $n_{H 0}$, Fig. 3 then reveals that at higher values of $n_{H 0}$, the non-adiabatic behaviour of $P_{1}$ already starts further inward at smaller solar distances $x$.

Furthermore, in Figs. 4 and 5, we show the polytropic index $\gamma_{1}(x)$ given in Eq. (34) as a function of $x$ for different values of $\Delta \alpha$ and $\Lambda$, respectively. As one can already see from Eqs. (31) and (34), the function $\gamma_{1}(x)$ reduces from

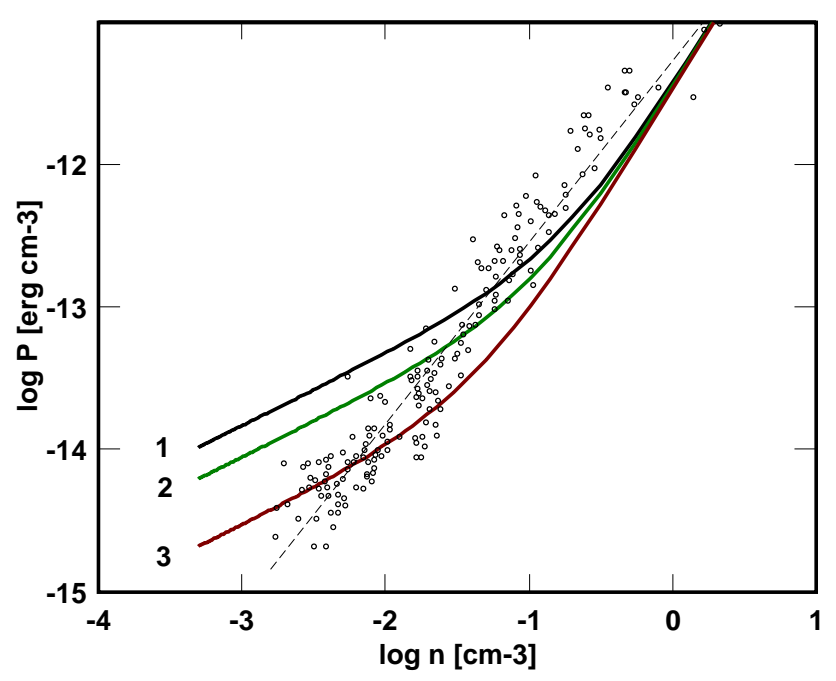

Fig. 3. Plotted is the logarithm of the solar wind pressure $P_{1}$ versus the logarithm of the density $\mathrm{n}_{1}$ at $\Delta \alpha=50$ and $T_{1,0}=5 \cdot 10^{4} \mathrm{~K}$ for different values of $\Lambda$, i.e. for $1: \Lambda=1 \cdot 10^{-3}, 2: \Lambda=2 \cdot 10^{-3}$, $3: \Lambda=3 \cdot 10^{-3}$. For comparison purposes data from the VOYAGER space probes are plotted as dots with a best fitting straight line (dashed curve), according to a polytropic index of $\gamma_{1}^{*}=1.28$ (see Whang, 1998).

its initial value of $\gamma_{1,0} \simeq \gamma=5 / 3$ to an asymptotic value of $\gamma_{1}(x \rightarrow \infty)=\gamma_{\infty}$, which depends neither on $\Delta \alpha$ nor $\Lambda$. The range of solar distances where $\gamma_{1}$ turns out to be between, say, 1.4 and 1.2 , i.e. clearly below the adiabatic value, is, however, fairly sensitive to both $\Lambda$ and $\Delta \alpha$. With parameter values $\Lambda=3 \cdot 10^{-3}$ and $\Delta \alpha=50$ one would obtain polytropic indices below 1.3 all the way from 5 AU outwards, as was already observed by VOYAGER-2 (see Whang, 1999).

\section{Average energy transfer between the $P(2)$ - and $P(1)$ - fluid}

In the preceding section we have used the hypothesis that waves driven by $\mathrm{P}(2)^{\text {'s }}$ energize solar wind protons and thereby eventually transfer a specific fraction of their initial pick-up energy per $\mathrm{P}(2)$, i.e. of $E_{i}=\frac{1}{2} m_{p} v_{w}^{2}$, to the solar wind background, i.e. to the $\mathrm{P}(1)^{\prime}$ s. We shall study which fraction of this initial $\mathrm{P}(2)$ - energy is eventually transfered to the $\mathrm{P}(1)^{\text {'s }} \mathrm{when}$ they finally leave the inner heliosphere, passing over the heliospheric termination shock. The net $\mathrm{P}(2)$-induced wave energy input to $\mathrm{P}(1)$ 's per unit volume and time, according to Eq. (12), is given by:

$$
\begin{gathered}
Q_{1}=-Q_{2}=-\left(\frac{\gamma}{\gamma-1}-\frac{v_{w}^{2}}{2 C_{2}}\right) \\
\operatorname{div}\left(P_{2} \boldsymbol{v}_{w}\right)+\left(\boldsymbol{v}_{w} \circ \nabla\right) P_{2}
\end{gathered}
$$

We may evaluate this expression here by assuming, as already done before, that $K T_{2}=m_{p} C_{2}$, as well as the solar 


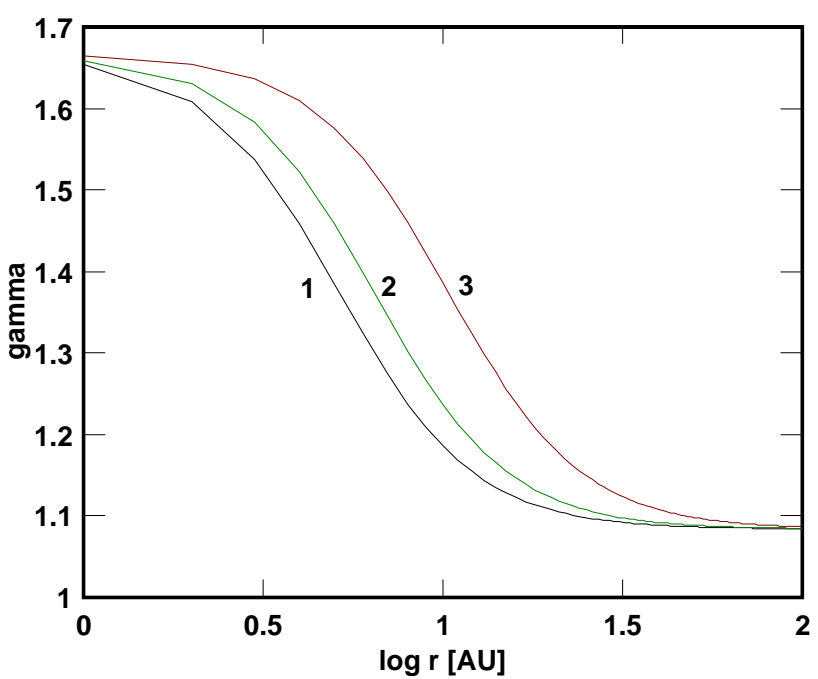

Fig. 4. Plotted is the polytropic index $\gamma_{1}$ versus the logarithm of the solar distance $r$ at $\Lambda=1 \cdot 10^{-3}$ and $T_{1,0}=5 \cdot 10^{4} \mathrm{~K}$ for different values of $\Delta \alpha=\alpha_{1}-\alpha_{2}$, i.e. for $1: \Delta \alpha=50,2: \Delta \alpha=30$, $3: \Delta \alpha=10$.

wind velocity $v_{w}$, are constants. Then the above expression evaluates to:

$$
\begin{array}{r}
Q_{1}=-\left(\frac{\gamma}{\gamma-1} K T_{2}-\frac{m_{p} v_{w}^{2}}{2}\right) \\
\quad \operatorname{div}\left(n_{2} \boldsymbol{v}_{w}\right)-K T_{2}\left(\boldsymbol{v}_{w} \circ \nabla\right) n_{2} .
\end{array}
$$

Keeping in mind that:

$\operatorname{div}\left(n_{2} \boldsymbol{v}_{w}\right)=n_{H} n_{1} \sigma_{e x} v_{w}$

and that $n_{2}=n-n_{1}$, with (see Eq. 30):

$$
n_{2}=n_{0} x^{-2}[1-\exp (-\Lambda(x-1))]
$$

then allows one to transform Eq. (36) into:

$$
\begin{aligned}
Q_{1}= & -\left(\frac{\gamma}{\gamma-1} K T_{2}-\frac{m_{p} v_{w}^{2}}{2}\right) \Lambda \frac{n_{0} v_{w}}{r_{0}}\left[x^{-2}(1-\Lambda(x-1)]\right. \\
& +K T_{2} \frac{v_{w} n_{0}}{r_{0}} \Lambda\left(x^{-2}+2 x^{-3}\right)
\end{aligned}
$$

which, in view of the fact that within our integration limits of $x \leq 100$, the quantity $\Lambda x \ll 1$ can be further simplified to:

$$
\begin{aligned}
Q_{1} & =-\Lambda \frac{n_{0} v_{w}}{r_{0}} \\
& \left\{\left(\frac{2 \gamma-1}{\gamma-1} K T_{2}-\frac{m_{p} v_{w}^{2}}{2}\right) x^{-2}+2 K T_{2} x^{-3}\right\} .
\end{aligned}
$$

With this expression for the $\mathrm{P}(2)$-induced energy input, one arrives at a total energy input per unit of time into a sector of the inner heliosphere, distending with a space angle $d \Omega$ from

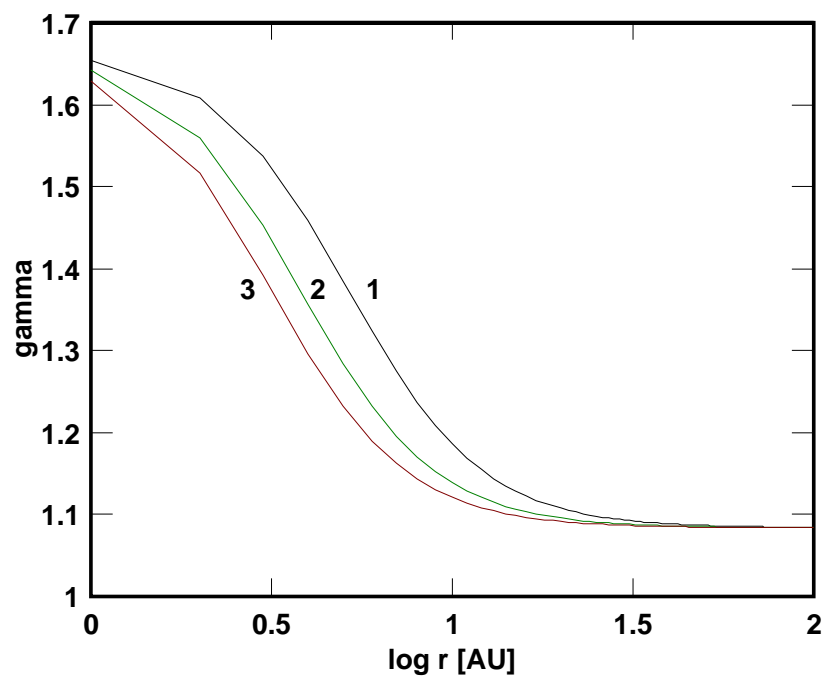

Fig. 5. Plotted is the polytropic index $\gamma_{1}$ versus the logarithm of the solar distance $r$ at $\Delta \alpha=50$ and $T_{1,0}=5 \cdot 10^{4} \mathrm{~K}$ for different values of $\Lambda$, i.e. for: $1: \Lambda=1 \cdot 10^{-3}, 2: \Lambda=2 \cdot 10^{-3}, 3: \Lambda=3 \cdot 10^{-3}$.

$r=r_{0}$ (i.e. inner boundary where no $\mathrm{P}(2)^{\prime}$ s are present) to $r=r_{s}=100 r_{0}$ (i.e. heliospheric shock location) given by:

$\Gamma_{1}=d \Omega \int_{r_{0}}^{r_{s}} r^{2} Q_{1} d r$

which, with the use of Eq. (40), takes the following form:

$$
\begin{gathered}
\Gamma_{1}=-r_{0}^{3} d \Omega \int_{1}^{x} x^{2} \Lambda \frac{n_{0} v_{w}}{r_{0}} \\
\left\{\left(\frac{2 \gamma-1}{\gamma-1} K T_{2}-\frac{m_{p} v_{w}^{2}}{2}\right) x^{-2}+2 K T_{2} x^{-3}\right\} d x,
\end{gathered}
$$

and thus can be simplified to:

$$
\begin{aligned}
\Gamma_{1}= & -\Lambda r_{0}^{2} n_{0} v_{w} d \Omega \int_{1}^{x} \\
& \left\{\left(\frac{2 \gamma-1}{\gamma-1} K T_{2}-\frac{m_{p} v_{w}^{2}}{2}\right)+2 K T_{2} x^{-1}\right\} d x .
\end{aligned}
$$

This finally can be evaluated to yield:

$$
\begin{aligned}
\Gamma_{1}= & -\Lambda r_{0}^{2} n_{0} v_{w} d \Omega \\
& \left\{\left(\frac{2 \gamma-1}{\gamma-1} K T_{2}-\frac{m_{p} v_{w}^{2}}{2}\right)(x-1)+2 K T_{2} \ln (x)\right\}
\end{aligned}
$$

For the outer boundary $x_{s} \simeq 100$ of the integration (i.e the location of the termination shock) this expression finally simplifies to:

$$
\Gamma_{1}=-\Lambda r_{0}^{2} n_{0} v_{w} d \Omega\left(\frac{2 \gamma-1}{\gamma-1} K T_{2}-\frac{m_{p} v_{w}^{2}}{2}\right) x_{s} .
$$


Now we want to compare this expression for $\Gamma_{1}$ with the total energy input $\Gamma_{i}$ into the same inner heliospheric solar wind sector per unit of time due to the total loading of the solar wind with freshly implanted PUI's of energy $E_{i}$ $=(1 / 2) m_{p} v_{w}^{2}$ at a local implantation rate $\beta_{e x}$ within the same space sector as considered above. For $\Gamma_{i}$ one thus obtains the following expression:

$\Gamma_{i}=d \Omega \int_{r_{0}}^{r_{s}} r^{2} \beta_{e x}(r)\left(\frac{1}{2} m_{p} v_{w}^{2}\right) d r$

Keeping in mind that the local PUI production rate can be expressed by $\beta_{e x}=\operatorname{div}\left(\xi n \boldsymbol{v}_{w}\right)$, then allows one to arrive at:

$\Gamma_{i}=\Lambda n_{0} r_{0}^{2} v_{w}\left[\frac{m_{p}}{2} v_{w}^{2}\right] d \Omega\left(x_{s}-1\right)$

The ratio $\Theta$ of the above energy inputs $\Gamma_{1}$ and $\Gamma_{i}$ taken from Eqs. (45) and (47) is thus given by:

$$
\begin{aligned}
\Theta & =\frac{\Gamma_{1}}{\Gamma_{i}}=\frac{-\Lambda r_{0}^{2} n_{0} v_{w} d \Omega\left(\frac{2 \gamma-1}{\gamma-1} K T_{2}-\frac{m_{p} v_{w}^{2}}{2}\right) x_{s}}{\Lambda n_{0} r_{0}^{2} v_{w} d \Omega\left[\frac{m_{p}}{2} v_{w}^{2}\right] x_{s}} \\
& =1-\frac{\frac{2 \gamma-1}{\gamma-1} K T_{2}}{\frac{m_{p}}{2} v_{w}^{2}}=1-\frac{\frac{2 \gamma-1}{\gamma-1}}{\frac{1}{2} M_{2}^{2}},
\end{aligned}
$$

where $M_{p u i}$ is the PUI Mach number defined by:

$M_{2}^{2}=\frac{\rho_{2} v_{w}^{2}}{P_{2}} \simeq \frac{1}{\alpha_{c}}\left(\frac{r_{s}}{r_{c}}\right)^{0.3}=2.24\left(\frac{r_{s}}{r_{c}}\right)^{0.3}$.

The above expression when evaluated for $\gamma=5 / 3$ then tells us that the above result can only reasonably well describe the $\mathrm{P}(1)-\mathrm{P}(2)$ two-fluid thermodynamics, if the $\mathrm{P}(2)$ Mach number fulfills the following relation:

$M_{2} \geq \sqrt[2]{7}=2.65$

As one can see in the result presented for $\Theta$ in Eq. (48) regarding the effectivity of the energy transfer from $\mathrm{P}(2)^{\prime}$ 's to $\mathrm{P}(1)^{\prime}$ s, the value of $\Lambda$, i.e. of $n_{H}$, does not play any role in this context. What counts, however, are the values of $\alpha_{1}$ and of $\alpha_{2}$, as one can see when rewriting Eq. (48) in the following form:

$\Theta=1-\frac{\frac{2 \gamma-1}{\gamma-1} K T_{2}}{\frac{m_{p}}{2} v_{w}^{2}}=\frac{\alpha_{2}-(2 \gamma-1) \alpha_{1}}{\alpha_{2}}$.

As one can conclude from the above relation, it is nec-

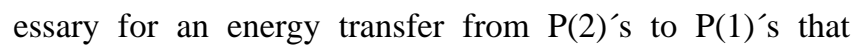
$\alpha_{2} \geq(7 / 3) \alpha_{1}$. For instance, for values like $\alpha_{2}=(8 / 3) \alpha_{1}$; $(9 / 3) \alpha_{1}$; (10/3) $\alpha_{1}$, , respectively, one could expect to have energy transfer ratios of $\Theta=0.125 ; 0.222 ; 0.3$.

\section{Concluding remarks}

We can state that whenever the solar wind system moves through a fractionally ionized interstellar medium, $\mathrm{P}(2)$ 's are automatically produced by ionization of neutral interstellar $\mathrm{H}$-atoms that penetrate into the supersonic region of the heliosphere. These ions, upon momentum-sharing with the solar wind at the $\mathrm{P}(2)$ - loading process, decelerate the wind. In addition, the original solar wind is modulated substantially in its dynamics and thermodynamics, when $\mathrm{P}(2)^{\prime}$ 's, as a separate suprathermal ion population, are mixed up with $\mathrm{P}(1)$ 's and at the same time are tied to a joint bulk velocity $v_{w}$. The solar wind is decelerated by about $10 \%$, depending on the density of the interstellar H-atoms (see appendix). In addition, the solar wind protons are polytropically heated by nonlinear wave-particle interactions induced by $\mathrm{P}(2)$-driven hydromagnetic waves, leading to a quasi-polytropic $\mathrm{P}(1)$ behaviour with distance-dependent polytropic indices $\gamma_{s}(x) \leq(5 / 3)$. A polytropic solar wind behaviour, with indices $\gamma_{s} \simeq 1.28$ in regions between 10 and $40 \mathrm{AU}$, as obtained in our calculations, is, in fact, confirmed by solar wind proton temperature measurements carried out with VOYAGER-2 (see Whang, 1999). By means of this nonlinear $\mathrm{P}(2)$-wave- $\mathrm{P}(1)$ energy coupling, about 10 to $20 \%$ of the intitial PUI injection energy $E_{i}$ is transfered to solar wind protons. The effective Mach numbers $M_{1,2}$ of the solar wind flow are reduced substantially to values of about 2 to 3 , which are associated primarily with the solar wind Mach number $M_{2}$ and are limited to $M_{2} \geq 2.65$. The two-fluid plasma mixture composed of $\mathrm{P}(1)$ 's and $\mathrm{P}(2)$ 's in many respects behaves like a mixture of a heavy and a light gas, except that the moment transfer terms are not of the type of the classical ones that are valid under collision-dominated conditions (see, for example, Braginskii, 1965; Burgers, 1969), but are by its nature wave-particle coupling terms.

Acknowledgement. The author is grateful for the financial support in the frame of the project FA.97/26-1 granted by the Deutsche Forschungsgemeinschaft.

Topical Editor G. Chanteur thanks two referees for their help in evaluating this paper.

\section{Appendix A}

In the following we come back to the Eq. (8) which was used in this paper and we want to evaluate in a more quantitative manner the expression

$P_{2} / \rho_{2}=\alpha_{c}\left(\frac{r_{s}}{r}\right)^{0.3} v_{w}^{2}$

First, we want to derive an expression for the solar wind deceleration as given in Eq. (3). In view of the fact that the relative $\mathrm{P}(2)$ ion abundance $\zeta(r)$ always remains small with respect to Eq. (3) (see Fahr and Rucinski, 1999), one can simplify Eq. (3) into the following form: 


$$
\begin{aligned}
v_{w}= & v_{w 0} \exp \left(\frac{2}{3} \int_{r_{0}}^{r} \frac{\xi}{r} d r\right) \\
& \exp \left(-\frac{4}{3} \int_{r_{0}}^{r} n_{H} \sigma_{e x}(1-\xi) d r\right) .
\end{aligned}
$$

From Eq. (7) in Fahr and Rucinski (1999) we learn that:

$\sigma_{e x} n_{H}=\frac{1}{1-\xi} \frac{\partial \xi}{\partial r}$

and obtain Eq. (A2) in the following form:

$v_{w}=v_{w 0} \exp \left(\frac{2}{3} \int_{r_{0}}^{r} \frac{\zeta}{r} d r\right) \exp \left(-\frac{4}{3}\left|\xi-\xi_{0}\right|\right)$.

Now taking into account that $\xi_{0}=\xi\left(r_{0}\right) \simeq 0$ and that for distances of relevance here, i.e. for $r \geq r_{c} \simeq 30 \mathrm{AU}$, the function $\xi=\xi(r)$ can be well approximated by:

$\xi(r)=\sigma_{e x} n_{H} r$

then yields Eq. (A4) in the following form:

$v_{w}=v_{w 0} \exp \left(-\frac{2}{3} \sigma_{e x} n_{H} r\right)$

which, aside from of the factor (2/3) instead of 1 , was also already found in an earlier work by Holzer (1972), Fahr (1973) and Lee (1997). Taking this result we then are led to the following expression for Eq. (A1):

$$
\begin{aligned}
& P_{2} / \rho_{2}=\alpha_{c}\left(\frac{r_{s}}{r}\right)^{0.3} v_{w}^{2} \\
& =\alpha_{c}\left(\frac{r_{s}}{r}\right)^{0.3}\left(1-\frac{4}{3} \sigma_{e x} n_{H} r\right) v_{w 0}^{2}
\end{aligned}
$$

With $\sigma_{e x} \simeq 10^{-15} \mathrm{~cm}^{2}$ and $n_{H}=n_{H \infty}=0.07 \mathrm{~cm}^{3}$, one then finds that between $r=r_{c}=30 \mathrm{AU}$ and $r=r_{s}=90 \mathrm{AU}$ the expression $P_{2} / \rho_{2}=K T_{2} / m_{p}$ given by Eq. (A6) only varies between the following values:

$0.58 v_{w 0}^{2} \geq P_{2} / \rho_{2} \geq 0.40 v_{w 0}^{2}$.

In view of this fairly mild variation over a large distance domain of the outer heliosphere we feel encouraged to approximate the temperature $T_{2}$ by:

$T_{2} \simeq 0.5\left(\frac{m_{p}}{K}\right) v_{w 0}^{2}[$ Kelvin $]$.

\section{References}

Baranov, V. B. and Malama, Y. G.: Modell of the solar wind interaction with the interstellar medium: Numerical solution of the self-consistent problem, J. Geophys. Res., 98, 15 157, 1993.

Baranov, V. B., and Malama, Y. G.: Effect of local interstellar medium hydrogen fractional ionization on the distant solar wind, J. Geophys. Res., 100, 14 755, 1995.
Baranov, V. B., Izmodenov, V., and Malama, Y. G.: On the distribution functions of $\mathrm{H}$-atoms in the problem of the solar wind interaction with the local interstellar medium, J. Geophys. Res., 103, 9575, 1998.

Braginskii, S. I.: "Transport processes in a plasma", Rev. Plasma Phys., 1, 205-311, 1965.

Bogdan, T. J., Lee, M. A., and Schneider, P.: Coupled quasi-linear wave damping and stochastic acceleration of pick-up ions in the solar wind, J. Geophys. Res., 96, 161-178, 1991.

Burgers, J. M.: "Flow equations of composite gases ", Appl. Math. Mech., Vol. II, Academic Press, New York, pp. 55-130, 1969.

Chalov, S. V. and Fahr, H. J.: Entropy generation at the multifluid solar wind termination shock producing anomalous cosmic ray particles, Planet. Space Sci., 43, 1035-1043, 1995.

Chalov, S. V. and Fahr, H. J.: A three-fluid model of the solar wind termination shock including a continuous production of anomalous cosmic rays, Astron. Astrophys., 311, 317-328, 1996.

Chalov, S. V. and Fahr, H. J.: The three-fluid structure of the particle modulated solar wind termination shock, Astron. Astrophys. 326, 860-869, 1997.

Chalov, S. V., Fahr, H. J., and Izmodenov, V.: Spectra of energized pick-up ions upstream of the heliospheric termination shock I. The role of Alfvénic turbulences, Astron. Astrophys., 304, 609, 1995.

Chalov, S. V., Fahr, H. J., and Izmodenov, V.: Spectra of energized pick-up ions upstream of the two-dimensional heliospheric termination shock. II. Acceleration by Alfvénic and by large-scale solar wind turbulences, Astron. Astrophys., 320, 659-671, 1997.

Coleman, P.: Turbulence, Viscosity, and Dissipation in the Solar Wind, Astrophys. J., 153, 371-388, 1968.

Fahr, H. J.: Non-Thermal Solar Wind Heating by Supra-Thermal Ions, Solar Physics, 30, 193, 1973.

Fahr, H. J. and Ripken, H. W.: The physics of the heliospheric interface and its implications for LISM diagnostics, Astron. Astrophys., 139, 551-554, 1984.

Fahr, H. J. and Ziemkiewicz, J.: The behavior of distant heliospheric pick-up ions and associated solar wind heating, Astron. Astrophys., 202-305, 295, 1988.

Fahr, H. J. and Fichtner, H.: The influence of pick-up ion-induced wave pressures on the dynamics of the mass-loaded solar wind, Solar Physics, 158, 353-363, 1995.

Fahr, H. J. and Rucinski, D.: Neutral interstellar gas atoms reducing the solar wind Mach number and fractionally neutralizing the solar wind, Astron. Astrophys., 350, 1071-1078, 1999.

Fahr, H. J. and Lay, G.: Remote diagnostic of the heliospheric termination shock using neutralized post shock pick-up ions as messengers, Astron. Astrophys., 356, 327-334, 2000.

Fahr, H. J., Kausch, T., and Scherer, H.: A 5-dimensional hydrodynamic approach to model the solar system-interstellar medium interaction, Astron. Astrophys., 357, 268, 2000.

Fahr, H. J. and Rucinski, D.: Modification of Properties and Dynamics of Distant Solar Wind Due to its Interaction with Neutral Interstellar Gas, Space Sci. Rev., 97, 407-412, 2001.

Fisk, L. A., Gloeckler, G., Zurbuchen, T. H., and Schwadron, N. A.: Ubiquitous statistical acceleration in the solar wind, in: ACE Symposium 2000, i (Ed) Mewaldt, R. A., American Institute of Physics, pp. 229-233, 2000.

Freund, H. P. and Wu, C. S.: Stability of spherical shell distributions of pick-up ions, J. Geophys. Res., 93, 14277, 1988.

Gray, P. C., Smith, C. W., Matthaeus, W. H., and Otani, N. F.: Heating the solar wind by pickup ion driven Alfvén ion cyclotron instability, Geophys. Res. Lett., 23, 113-116, 1996. 
Gary, S. P., and Feldman, W. C.: A second-order theory for $\boldsymbol{k} \| \boldsymbol{B}_{O}$ electromagnetic instabilities, Phys. Fluids, 21, 72-80, 1978.

Hartle, R. E. and Wu, C. S.: Effects of electrostatic instabilities on planetary and interstellar ions in the solar wind, J. Geophys. Res., 78, 5802, 1973.

Holzer, T. E.: Interaction of the solar wind with the neutral component of the interstellar gas, J. Geophys. Res., 77, 5407, 1972.

Holzer, T. E. and Leer, E.: Conductive solar wind models in rapidly diverging flow geometries, J. Geophys. Res., 85, 4665-4679, 1980.

Huddleston, D. E. and Johnstone, A. D.: Relationship between wave energy and free energy from pick-up ions in the Comet Halley environment, J. Geophys. Res., 97, 12 217, 1992.

Isenberg, P. A.: Interaction of the solar wind with interstellar neutral hydrogen: Three fluid model, J. Geophys. Res., 91, 9965-9972, 1986.

Kennel, C. F. and Engelmann, F.: Velocity space diffusion from weak plasma turbulence in a magnetic field, Phys. Fluids, 9, 2377, 1966.

Lee, M. A.: Effects of cosmic rays and interstellar gas on the dynamics of a wind, in: Stellar Winds, (Eds) Jokopii, J. R., Sonett, C. P., and Giampapa M., Tucson, Arizona, University of Arizona, pp. 857-886, 1997.

Lee, M. A. and Ip, W. H.: Hydrodynamic wave excitation by ionised interstellar hydrogen and helium in the solar wind, J. Geophys. Res., 92, 11 041-11 052, 1987.

Le Roux, J. A. and Fichtner, H.: A self-consistent determination of the heliospheric termination shock structure in the presence of pick-up, anomalous, and galactic cosmic ray protons, J. Geophys. Res., 102, 17 365-17380, 1997.

Mattheaus, W., Oughton, S., Pontius, D., and Zhou, Y.: Evolution of energy-containing turbulent eddies in the solar wind, J. Geophys. Res., 99, 19267-19287, 1994.

Parker, E. N.: Dynamical properties of stellar coronas and stellar winds. II. Integration of the heat flow equation, Astrophys. J., 139, 93, 1964.

Pauls, H. L., Zank, G. P., and Williams, L. L.: Interaction of the solar wind with the local interstellar medium, J. Geophys. Res., 100, 21,595, 1995.

Richardson, J. D., Paularena, K. I., Lazarus, A. J., and Belcher, J. W.: Radial evolution of the solar wind from IMP 8 to Voyager 2, Geophys. Res. Lett., 22, 325, 1995.
Ripken, H. W. and Fahr, H. J.: Modification of the local interstellar gas properties in the heliospheric interface, Astron. Astrophys., 122, 181-192, 1983.

Smith, C. W., Matthaeus, W. H., Zank, G. P., Ness, N. F., Oughton, S., and Richardson, J. D.: Heating of the low latitude solar wind by dissipation of turbulent magnetic fluctuations, J. Geophys. Res., 106, 8253, 2001.

Wang, C. J., Richardson, D., and Gosling, J. T.: Slowdown of the Solar Wind in the Outer Heliosphere and the Interstellar Neutral Hydrogen Density, Geophys. Res. Lett., 27, 2429-2432, 2000.

Whang, Y. C.: Solar Wind in the Distant Heliosphere, J. Geophys. Res., 103, 17 419-17 428, 1998.

Whang, Y. C., Lu, J. Y., and Burlaga, L. F.: The termination shock 1979-1996, J. Geophys. Res., 104, 28 255, 1999.

Williams, L. L., Zank G. P., and Matthaeus, W. H.: Dissipation of pickup-induced waves: A solar wind temperature increase in the outer heliosphere?, J. Geophys. Res., 100, 17 059-17 098, 1995.

Winske, D. and Leroy, M. M.: Diffuse ions produced by electromagnetic ion beam instabilities, J. Geophys. Res., 89, 26732688, 1984.

Wu, C. S. and Davidson, R. C.: Electromagnetic instabilities produced by neutral-particle ionization in interplanetary space, J. Geophys. Res., 77, 5399, 1972.

Wu, F. M. and Judge, D. L., Temperature and flow velocity of the interplanetary gases along solar radii, Astrophys. J., 231, 594605, 1979.

Zank, G. P.: Interaction of the solar wind with the local interstellar medium: A theoretical perspective, Space Sci. Rev., 89, 413, 1999.

Zank, G. P., Pauls, H. L., Williams, L. L., and Hall, D. T.: Interaction of the solar wind with the local interstellar medium: A multifluid approach, J. Geophys. Res., 101, 21 639, 1996a.

Zank, G. P., Matthaeus, W. H., and Smith, C. W.: Evolution of turbulent magnetic fluctuation power with heliospheric distance, J. Geophys. Res., 101, 17 081, 1996 b.

Zank, G. P., Webb, G. M., and Donohue, D. J.: Particle injection and the structure of energetic particle-modified shocks, Astrophys. J., 406, 67-91, 1993.

Zhou, Y. and Matthaeus, W. H.: Models of inertial range spectra of interplanetary magneto-hydrodynamic turbulence, J. Geophys. Res., 95, $14881,1990$. 